\title{
Degradation of methylene blue by three- dimensionally ordered macroporous titania
}

\author{
Supporting Information
}

Manuscript Number:

Madhavi Srinivasan ${ }^{*}$ and Tim White

Nanyang Technological University, School of Materials Science and Engineering, Blk N4.1-01-30, 50 Nanyang Avenue, Singapore 639798

*Corresponding author: Tel:(65) 67904606, Fax: (65) 67909081, email: madhavi@ntu.edu.sg 


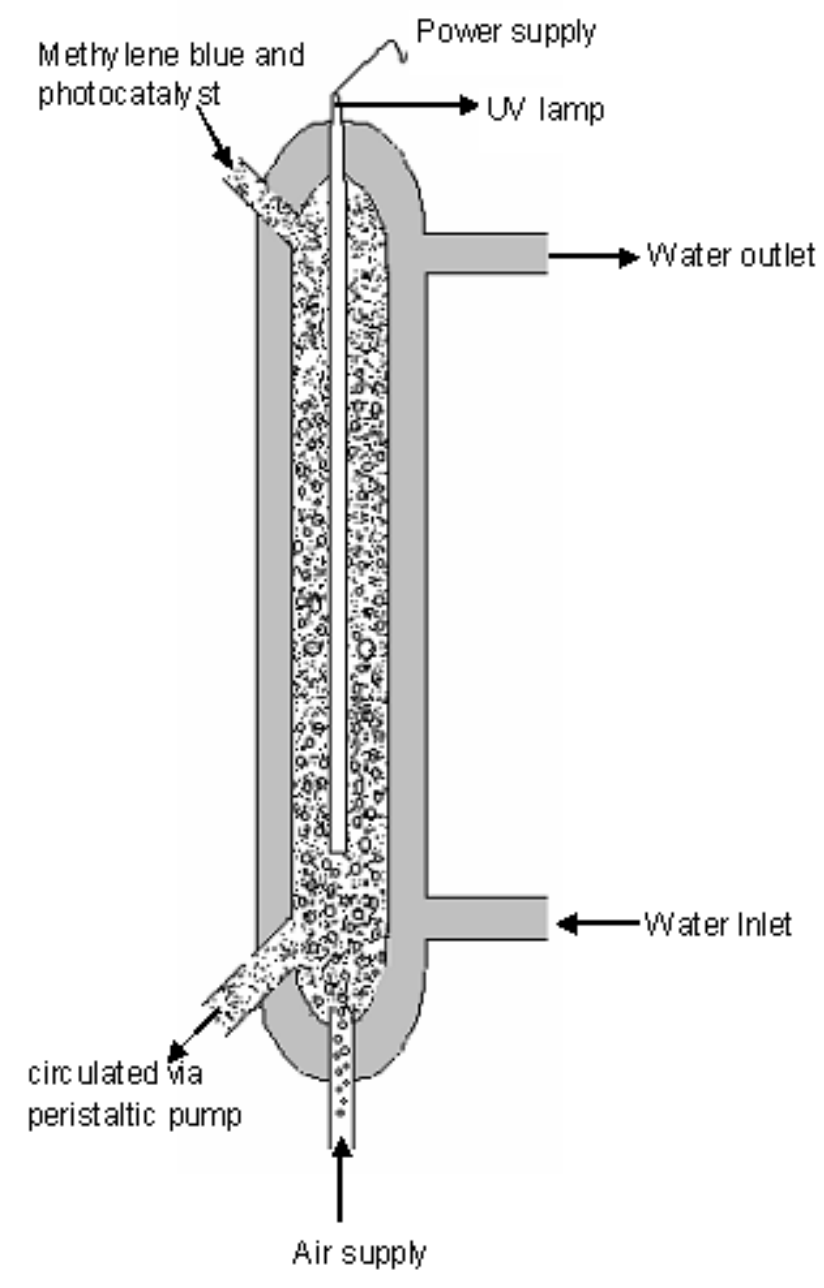

S1: Schematic of the reactor used for photocatalytic degradation of methylene blue. 


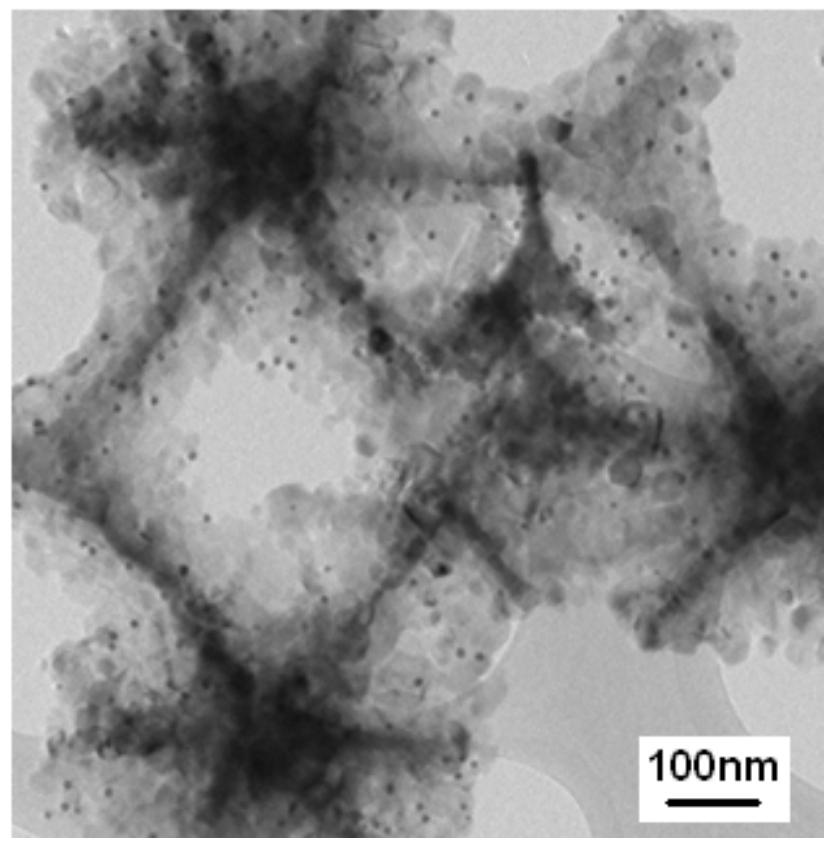

S2: Gold nanocrystals deposited on the macroporous titania walls (Au1) 

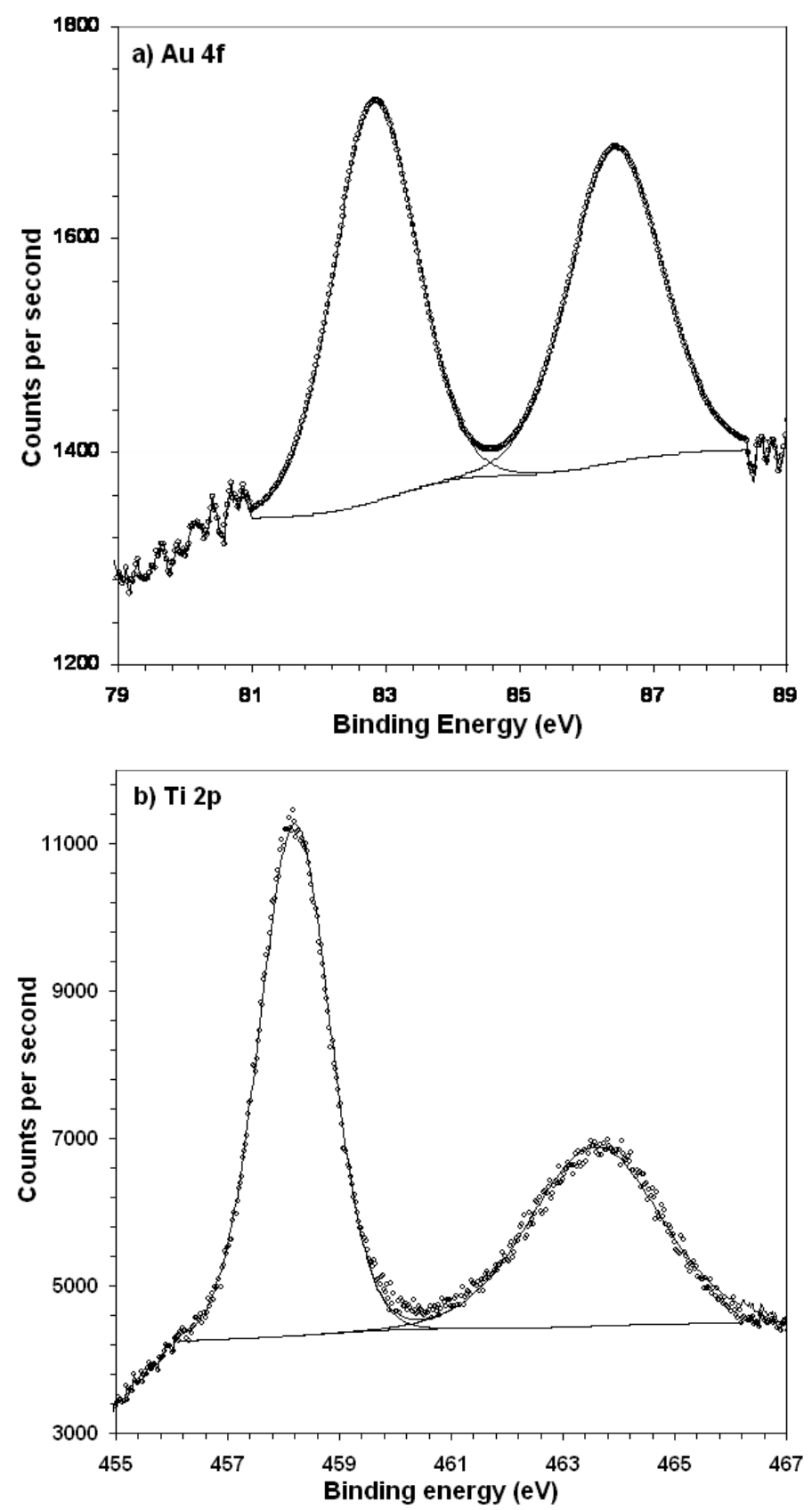

S3: High resolution curve fitted (solid lines) X-ray photoelectron spectra of a) Au4f and b) Ti2p in gold deposited 3DOM titania (Au1). 


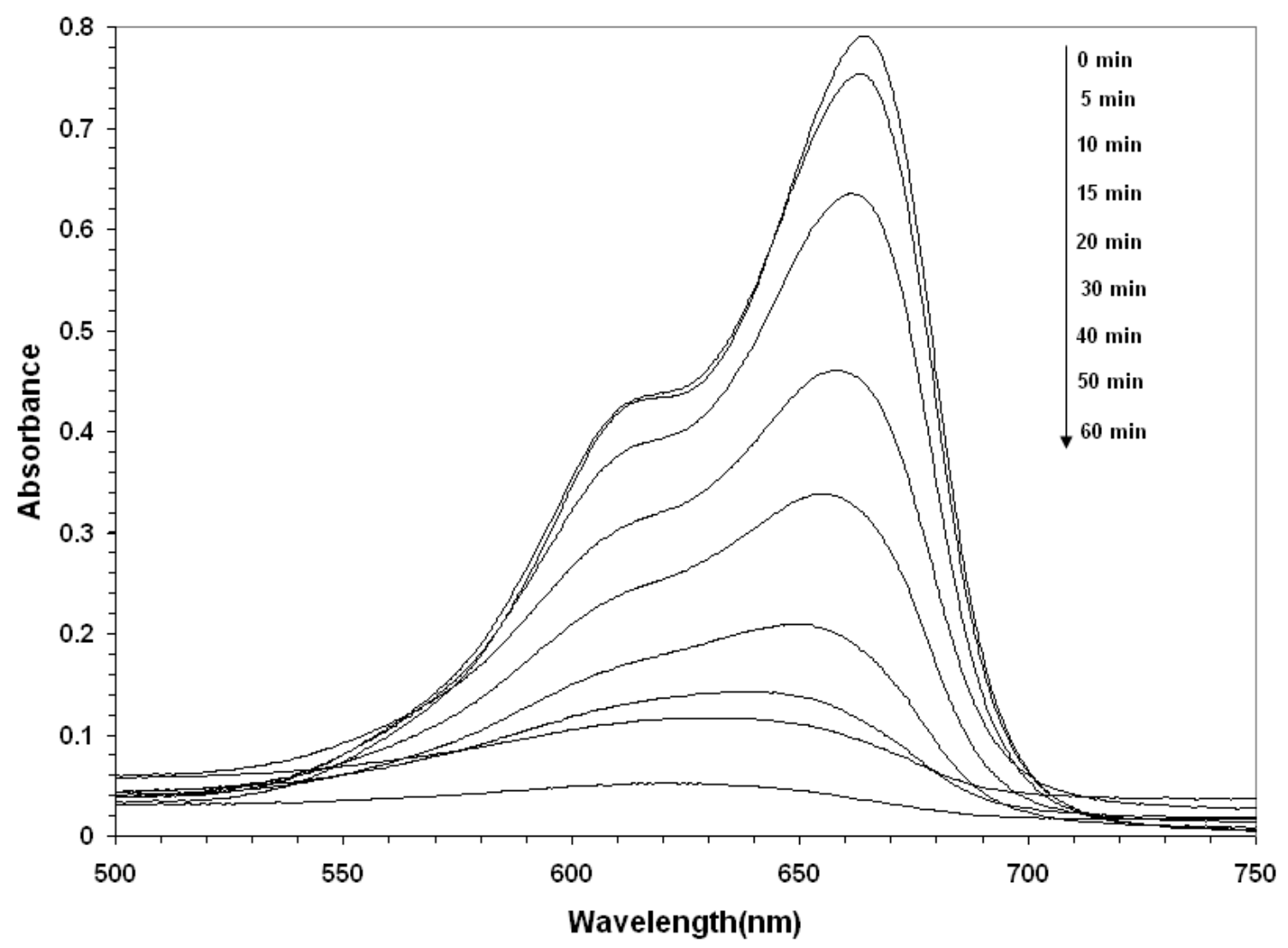

S4: Variation in the UV-visible absorption spectra of 3DOM titania (MT5) as a function of UV-irradiation time. 


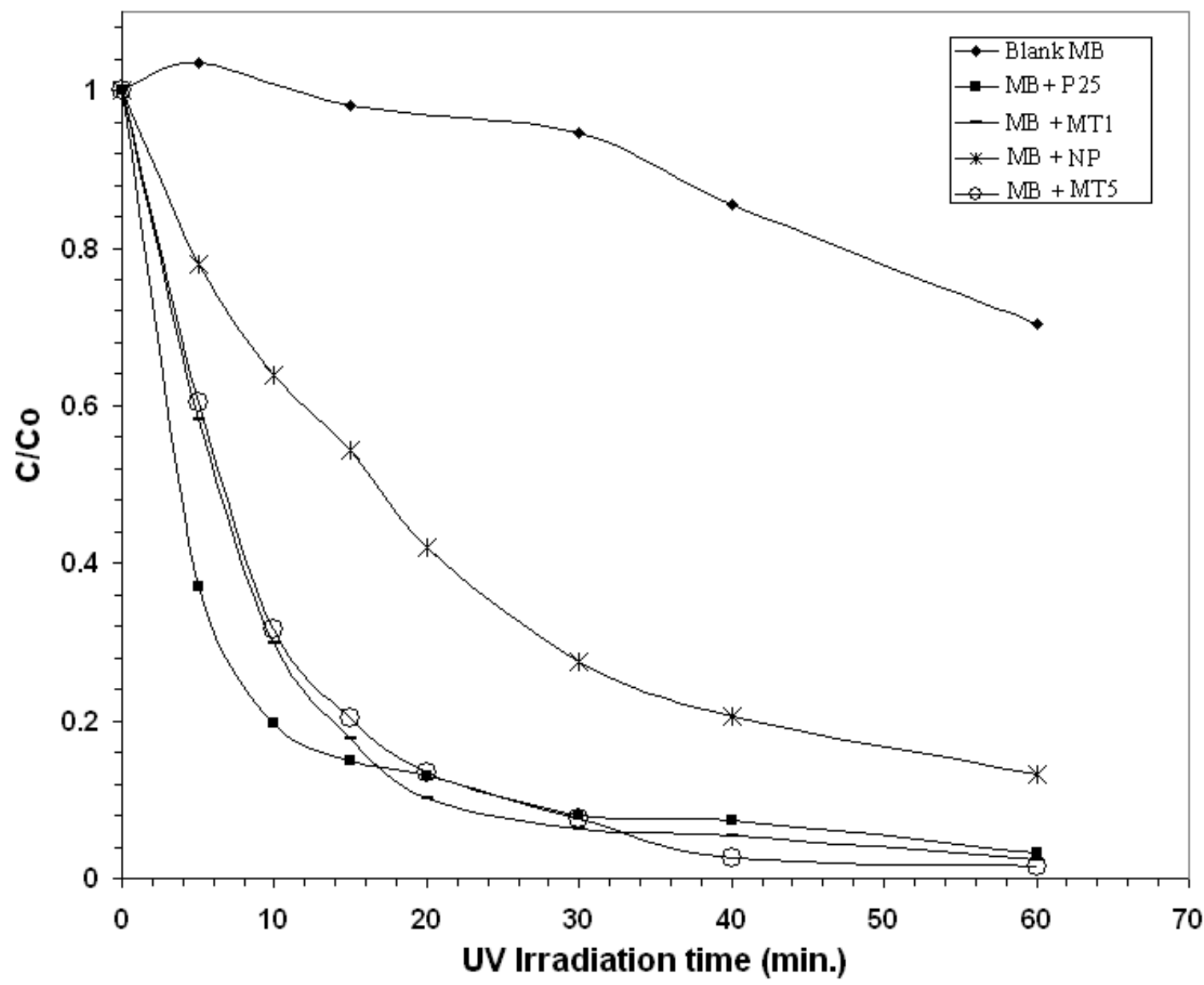

S5: Variation in the concentration of MB with UV-irradiation time using protocol 1 (5 minutes ultrasonic treatment) with different photocatalysts. 


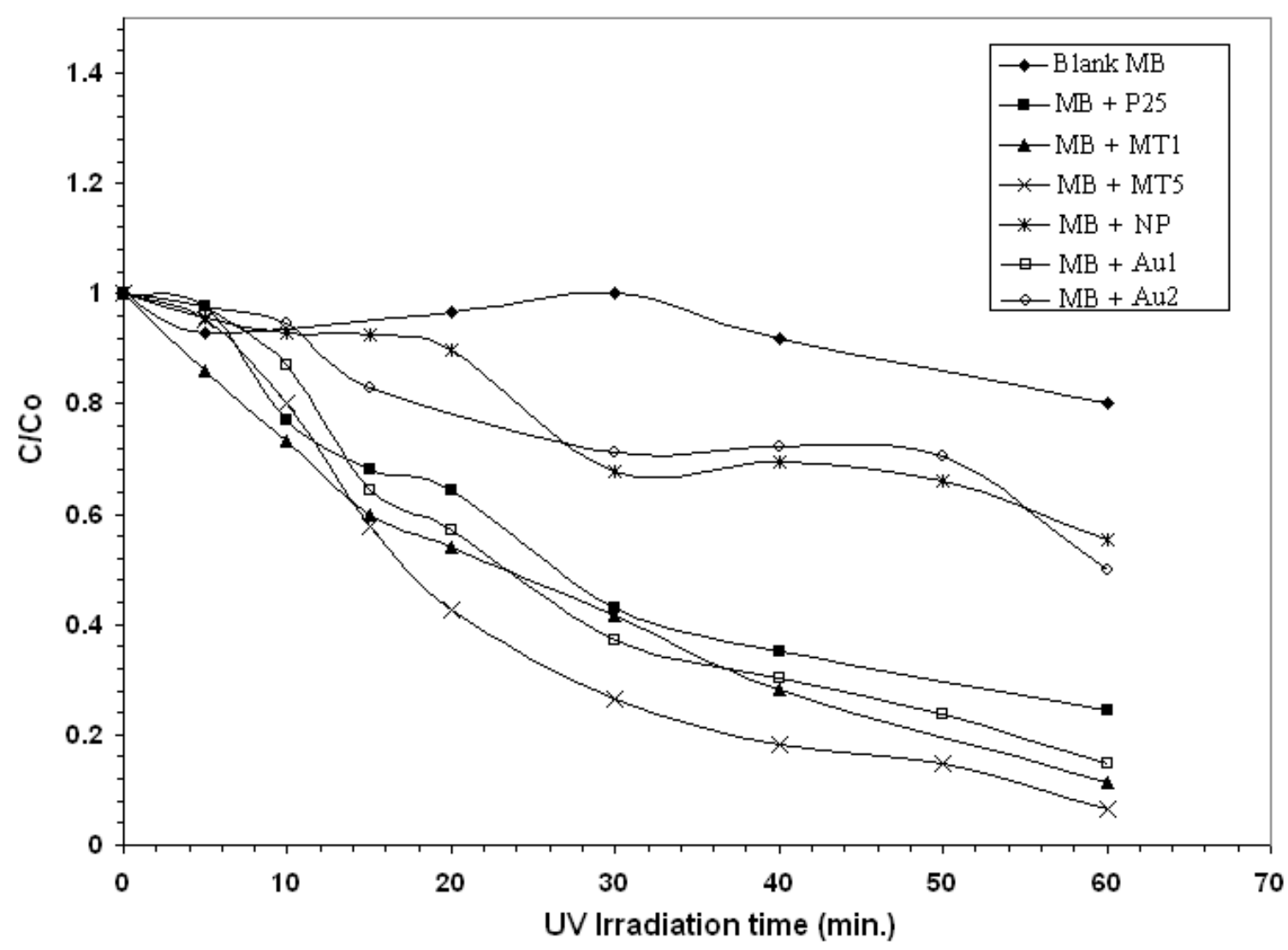

S6: Variation in the concentration of MB with UV-irradiation time using protocol 2 (30 minutes stirring in the dark) with different photocatalysts. 